\title{
CARACTERIZAÇÃO DE DOIS ESTRATOS DA VEGETAÇÃO EM UMA ÁREA DE CERRADO NO MUNICÍPIO DE BROTAS, SP, BRASIL
}

\author{
Giselda Durigan ${ }^{1}$ \\ Dulcelaine L. Lopes Nishikawa ${ }^{2}$ \\ Elektra Rocha ${ }^{2}$ \\ Éliton Rodrigo da Silveira ${ }^{2}$ \\ Fabiana Marise Pulitano ${ }^{2}$ \\ Luciano Bonatti Regalado ${ }^{2}$ \\ Mariana Aparecida Carvalhaes ${ }^{2}$ \\ Patrícia Amaral Paranaguá ${ }^{2}$ \\ Victor Eduardo Lima Ranieri ${ }^{3}$
}

Recebido em 20/03/2001. Aceito em 10/12/2001.

\begin{abstract}
RESUMO - (Caracterização de dois estratos da vegetação em uma área de cerrado no município de Brotas, SP, Brasil). Caracterizaram-se dois estratos da vegetação de uma área de cerca de 300 ha de cerrado stricto sensu, localizada no município de Brotas, SP, quanto à sua estrutura e diversidade, pelo método de parcelas. Para amostragem do estrato arbóreo (diâmetro mínimo de $5 \mathrm{~cm}$, medido a $50 \mathrm{~cm}$ acima do nível do solo), foi amostrada área total de $5000 \mathrm{~m}^{2}(250 \times 20 \mathrm{~m})$, divididos em 50 parcelas contíguas de $10 \times 10 \mathrm{~m}\left(100 \mathrm{~m}^{2}\right)$. Para o estrato inferior (altura < $50 \mathrm{~cm}$ e diâmetro do caule inferior a $5 \mathrm{~cm}$ ), a área total de amostragem foi de $500 \mathrm{~m}^{2}$, distribuídos em 50 subparcelas de $10 \times 1 \mathrm{~m}\left(10 \mathrm{~m}^{2}\right)$, demarcadas dentro de cada uma das parcelas maiores. Determinou-se a cobertura do estrato arbóreo pelo método de interceptação de linhas e o índice de agregação das espécies e da comunidade, segundo Payandeh. Foram identificadas, no total, 78 espécies ( 36 famílias). O estrato arbóreo apresentou densidade de $1150 \mathrm{ind} / \mathrm{ha}, 7,2 \mathrm{~m}^{2} /$ ha de área basal e $19,6 \%$ de cobertura. Foram amostradas, neste estrato, 44 espécies (27 famílias), com $\mathrm{H}^{\prime}=3,02$ e os indivíduos da comunidade como um todo apresentaram-se agregados $(\mathrm{P}=4,26)$. As espécies de maior valor de importância no estrato superior, em ordem decrescente, foram: Acosmium subelegans, Aspidosperma tomentosum, Eriotheca gracilipes, Styrax ferrugineus e Tabebuia ochracea. No estrato inferior, com densidade de $10.220 \mathrm{ind} / \mathrm{ha}$, foram amostradas 61 espécies (32 famílias), destacando-se, em ordem decrescente de densidade relativa: Campomanesia adamantium, Bromelia balansae, Attalea geraensis, Xylopia aromatica e Ocotea pulchella. A comunidade apresentou-se também agregada no estrato inferior $(\mathrm{P}=3,89)$ e obteve-se índice de diversidade (H') de 3,32. Algumas espécies abundantes no estrato arbóreo, como Styrax ferrugineus, Eriotheca gracilipes, Piptocarpha rotundifolia e Annona crassiflora, não foram amostradas no estrato inferior, sugerindo a existência de restrições aos processos naturais de reprodução e regeneração. Essas espécies podem estar correndo o risco de extinção local.
\end{abstract}

Palavras-chave - Cerrado stricto sensu, fitossociologia, estratificação, diversidade

\footnotetext{
${ }^{1}$ Instituto Florestal, Estação Experimental de Assis, Caixa Postal 104, 19800-000, Assis, SP. giselda@femanet.com.br.

2 Programa de Pós-graduação em Ciências da Engenharia Ambiental, CRHEA - Centro de Recursos Hídricos e Ecologia Aplicada , USP, São Carlos.

${ }^{3}$ Programa de Pós-graduação em Hidráulica e Saneamento, Depto. de Hidráulica e Saneamento, EESC-USP, São Carlos
} 


\begin{abstract}
Characterization of two strata of cerrado vegetation at Brotas, SP, Brazil). Two strata of cerrado stricto sensu vegetation were surveyed, in a fragment covering an area of 300 ha, at Brotas, SP, Brazil. The sampling area had $5000 \mathrm{~m}^{2}(250 \times 20 \mathrm{~m})$. The upper stratum (diameter $50 \mathrm{~cm}$ above ground $\geq 5 \mathrm{~cm}$ ) was surveyed in 50 plots, $100 \mathrm{~m}^{2}$ each, and the lower stratum (diameter $<5 \mathrm{~cm}$ and height $<50 \mathrm{~cm}$ ) was surveyed in 50 sub-plots, $10 \mathrm{~m}^{2}$ each, for phytosociological analysis. Crown cover was estimated by the line interception method and the aggregation index of Payandeh was also calculated, for each species and for the community as a whole. Trees, shrubs, palms and herbs were identified, comprising 78 species (36 families). The upper stratum had $1150 \mathrm{ind} / \mathrm{ha}$, basal area of $7.2 \mathrm{~m}^{2} /$ ha and crown cover of $19.6 \%$. In this stratum, 44 species ( 27 families) were surveyed $\left(H^{\prime}=3.02\right)$ and the community was aggregated $(\mathrm{P}=4.26)$. The most important species (decreasing importance value) were: Acosmium subelegans, Aspidosperma tomentosum, Eriotheca gracilipes, Styrax ferrugineus and Tabebuia ochracea. The density of the lower stratum was 10,220 ind/ha, the community was also aggregated $(\mathrm{P}=3.89)$ and the diversity index $\left(\mathrm{H}^{\prime}=3.32\right)$ was higher than in the upper stratum. From the 61 species surveyed (32 families), the most important in decreasing order of relative density were: Campomanesia adamantium, Bromelia balansae, Attalea geraensis, Xylopia aromatica e Ocotea pulchella. Some of the most abundant species of the upper layer, such as Styrax ferrugineus, Eriotheca gracilipes, Piptocarpha rotundifolia and Annona crassiflora, were not found in the lower stratum. It is suggested that the regeneration process was interrupted and such populations may be locally threatened.
\end{abstract}

Key words - Cerrado stricto sensu, phytosociology, stratification, diversity

\section{Introdução}

A região de domínio do bioma Cerrado compreende uma extensa área contínua nos Estados de Goiás, Bahia, Minas Gerais e Mato Grosso e algumas penínsulas e áreas disjuntas que se estendem por outros estados (Eiten, 1972). No Estado de São Paulo, o Cerrado apresenta-se na forma de manchas dispersas, que ocupavam originalmente cerca de $14 \%$ do território (São Paulo, 1997). Em 1962, segundo Borgonovi \& Chiarini (1965), a vegetação de Cerrado cobria $33.929 \mathrm{~km}^{2}$. Onze anos depois, Serra Filho et al. (1974) estimaram essa cobertura em apenas $10.388 \mathrm{~km}^{2} \mathrm{e}$ Kronka et al. (1998), com imagens de 1992, encontraram área total remanescente de apenas $2.379 \mathrm{~km}^{2}$ de Cerrado, comprovando a rápida destruição desse bioma no Estado.

A vegetação de Cerrado compreende um gradiente de fitofisionomias correspondente a um gradiente de biomassa: campo sujo, campo cerrado, cerrado e cerradão. Mapear as fitofisionomias separadamente é difícil, seja por não haver nitidez na separação entre elas, seja porque as fitofisionomias se alteram ao longo do tempo em um mesmo local, em função da freqüência e intensidade de impactos como o fogo e o pastoreio.
Chiarini \& Coelho (1969), através da interpretação de fotografias aéreas de 1962, encontraram predominância da fisionomia cerrado (75 $\%)$ sobre as fisionomias de campo (16\%) e cerradão (9\%). Kronka et al. (1998), mapeando os remanescentes a partir de imagens de satélite, encontraram $68,9 \%$ de cerrado, $30,5 \%$ de cerradão e $0,6 \%$ de campo cerrado.

$\mathrm{Na}$ região de Brotas (SP), onde se insere a área de estudo, predominam as fisionomias campestres: campo cerrado e cerrado.

A vegetação de Cerrado no Estado de São Paulo tem sido objeto de diversos estudos, concentrados nas décadas de 70 e 80, mapeados por Durigan et al. (1999). Leitão Filho (1992), analisando trabalhos publicados e coleções de herbário, publicou "A flora arbórea dos cerrados do Estado de São Paulo". O que se verifica é que, com raras exceções (p. ex. Mantovani, 1987 e Durigan et al. 1999), esses estudos restringem-se ao estrato arbóreo. Estudos fitossociológicos envolvendo diferentes estratos da vegetação de cerrado são inexistentes.

O presente trabalho teve como objetivo a caracterização detalhada de dois estratos da vegetação em uma área de cerrado stricto sensu, visando a compreensão da estrutura e diversidade 
da comunidade vegetal, bem como aspectos da ecologia das populações, entre os quais a distribuição espacial dos indivíduos e a regeneração das espécies.

\section{Material e métodos}

Área de estudo - trata-se de um fragmento com área aproximada de 300 ha (Fig. 1), de propriedade da Universidade de São Paulo, situado à margem esquerda da Represa do Lobo, no município de Brotas, SP (22 $11^{\circ}$ 'S e $\left.47^{\circ} 54^{\prime} \mathrm{W}\right)$.

A altitude média da área é de $710 \mathrm{~m}$ e o solo, segundo Freire et al. (1980), é do tipo Areia Quatzosa Vermelho-Amarela Câmbica Distrófica Álica A fraco. O clima, segundo a classificação de Köppen, é Cwa: quente, de inverno seco, para clima tropical com verão úmido e inverno seco, precipitação média anual entre 1400 e 1500mm (Messina, 1998).

Levantamento fitossociológico - optou-se pela amostragem pelo método de parcelas, para os dois estratos, segundo os seguintes procedimentos e critérios:

Estrato superior: amostrou-se área contínua de $5000 \mathrm{~m}^{2}(250 \times 20 \mathrm{~m})$, localizada na porção central do fragmento, estando as parcelas distantes pelo menos $100 \mathrm{~m}$ da margem do reservatório. A área de amostragem foi subdividida em 50 parcelas de $10 \times 10 \mathrm{~m}\left(100 \mathrm{~m}^{2}\right)$, em que foram identificados e medidos altura e diâmetro de todos os indivíduos com DAJ (diâmetro à altura do joelho, medido a $50 \mathrm{~cm}$ acima do nível do solo) igual ou superior a $5 \mathrm{~cm}$.

Estrato inferior: demarcaram-se 50 sub-parcelas de $10 \times 1 \mathrm{~m}\left(10 \mathrm{~m}^{2}\right)$, cada uma no interior de uma das parcelas de amostragem do estrato superior, em que foram identificados e medida a altura de todos os indivíduos a partir de $50 \mathrm{~cm} \mathrm{e}$ com DAJ inferior a $5 \mathrm{~cm}$.

A identificação das espécies foi feita com o auxílio de chaves de identificação (Mantovani et al. 1985; Batalha \& Mantovani 1999) e de bi- bliografia especializada. Material cuja identificação no campo não foi possível foi herborizado e identificado posteriormente por comparação com material de herbário e consulta a especialistas. Este material encontra-se depositado na coleção botânica da Estação Experimental de Assis, do Instituto Florestal.

Os cálculos fitossociológicos de freqüência, densidade e dominância seguiram MüllerDombois \& Ellenberg (1974) e geraram os valores de importância (VI) e cobertura (VC) para as espécies. Para cálculos de dominância, embora a área basal seja medida, por definição, a 1,30 m acima do nível do solo, adotou-se a medição de diâmetro do caule a apenas $50 \mathrm{~cm}$ do solo, pois a tortuosidade e abundante ramificação das árvores de cerrado dificultam tremendamente as medições à altura do peito.

Para efeito de comparação, efetuou-se a estimativa de densidade da comunidade pelo método de quadrantes (Cottam \& Curtis, 1956), a partir da média das distâncias árvore-ponto, com um total de 50 pontos amostrados (200 indivíduos), no interior da área de amostragem por parcelas.

Diversidade - Calcularam-se os índices de diversidade de Shannon (Pielou, 1975) e equabilidade (Pielou, 1969), segundo as fórmulas:

$$
\begin{aligned}
& \mathrm{H}^{\prime}=-\sum_{i=1}^{n} \mathrm{Pi} \ln \mathrm{Pi} \\
& \mathrm{E}=\mathrm{H}^{\prime} / \mathrm{H}_{\text {máx }}^{\prime}
\end{aligned}
$$

Onde:

$\mathrm{H}^{\prime}=$ Índice de diversidade de Shannon

$\mathrm{E}=$ Equabilidade

$\mathrm{Pi}=$ ni $/ \mathrm{N}$

ni $=$ número de indivíduos da espécie $\mathrm{i}$

$\mathrm{N}=$ número total de indivíduos amostrados

$\ln =$ logaritmo neperiano

$\mathrm{E}=$ equabilidade 


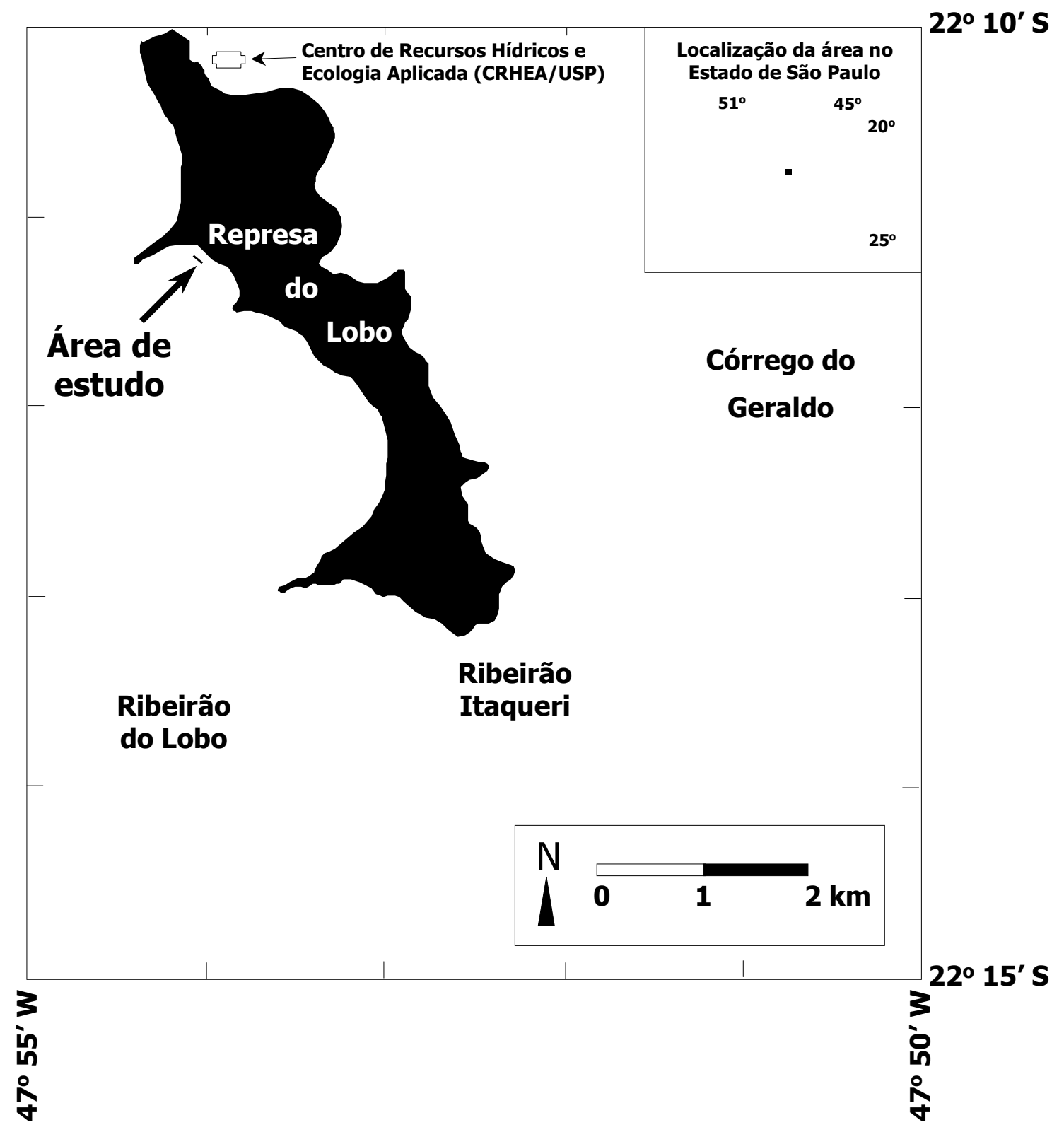

Figura 1. Localização da área de estudo, município de Brotas, Estado de São Paulo. 
Similaridade - Efetuaram-se cálculos de similaridade, utilizando-se o índice de Jaccard (Magurran, 1988), entre a área estudada e outras áreas de cerrado na região e entre os dois estratos amostrados, segundo a fórmula:

$\mathrm{Cj}=\mathrm{c} /(\mathrm{a}+\mathrm{b}-\mathrm{c})$,

Onde:

$\mathrm{Cj}=$ índice de similaridade

$\mathrm{a}=$ número total de espécies presentes no local A

$\mathrm{b}=$ número total de espécies presentes no local B

$\mathrm{c}=$ número de espécies comuns aos locais $\mathrm{A}$ e B.

Distribuição das populações - Para verificar o padrão de distribuição espacial dos indivíduos de cada espécie e da comunidade como um todo, adotou-se o Índice de Agregação de Payandeh (1970), expresso pela fórmula:

$$
P=\operatorname{Var} n i / \overline{n i}
$$

Onde:

$\mathrm{P}$ = índice de agregação

ni $=$ número de indivíduos da espécie i por parcela

$\overline{\mathrm{ni}}=$ número médio de indivíduos da espécie $\mathrm{i}$ por parcela

Valores de $\mathrm{P}$ superiores a 1,5 indicam distribuição agregada; $\mathrm{P}$ variando de 1,0 a 1,5 corresponde a distribuição aleatória e $\mathrm{P}$ inferior a 1,0 indica distribuição regular. Para espécies com um único indivíduo amostrado, não foi calculado o valor de P.

Cobertura das copas - Para estimativa da cobertura das copas (porcentagem do terreno coberta pela projeção das copas do estrato arbóreo), adotou-se o método de interceptação de linhas (Canfield, 1941), medindo-se a projeção das copas sobre uma trena, em uma ex- tensão de 100 m no interior da área de amostragem.

\section{Resultados e discussão}

Foram identificadas, no total, 78 espécies vegetais, pertencentes a 36 famílias (Tab. 1), sendo 48 árvores, 17 arbustos, 7 subarbustos, 2 palmeiras acaules e 3 espécies herbáceas. Do total de espécies amostradas, apenas 27 ocorreram nos dois estratos. No estrato superior (DAJ $\geq 5 \mathrm{~cm}$ ) foram amostradas 44 espécies, pertencentes a 27 famílias (Tab. 2).

Em outras áreas de amostragem do estrato arbóreo de vegetação de cerrado na mesma região, Toledo Filho et al. (1989) encontraram 103 espécies em Mogi-Mirim, Pagano et al. (1989) encontraram 125 espécies em Corumbataí, Castro (1987), em Santa Rita do Passa Quatro, registrou 110 espécies e, em Itirapina, Giannotti (1988) encontrou 118 espécies, em área de transição entre cerrado e mata ciliar. Durigan et al. (1994), em área submetida a incêndios freqüentes, registraram apenas 44 espécies no estrato arbóreo. Em comparação com essas outras áreas, quanto ao número de espécies arbóreas amostradas, a riqueza de espécies na área estudada foi baixa, comparável apenas à área fortemente impactada por incêndios freqüentes em Itirapina.

Analisando-se a similaridade florística do estrato arbóreo entre a área estudada e outras áreas de cerrado na região, pelo índice de Jaccard, encontra-se 25\% com Mogi-Guaçu (Mantovani et al., 1985) 23\% com Corumbataí (Cesar et al. 1988) e $29 \%$ com área freqüentemente queimada no município de Itirapina (Durigan et al. 1994). Considerandose que $25 \%$ é o limite mínimo para duas serem consideradas floristicamente semelhantes (Müller-Dombois \& Ellenberg, 1974), pode-se afirmar que os valores de similaridade encontrados entre a área estudada e outras áreas de cerrado na região são baixos. 
Tabela 1. Espécies vegetais presentes em área de Cerrado stricto sensu, Brotas, SP. S: espécie amostrada no estrato superior; I: espécie amostrada no estrato inferior.

\begin{tabular}{|c|c|c|c|c|}
\hline FAMÍLIA & ESPÉCIE & HÁBITO & $\mathrm{S}$ & I \\
\hline \multirow[t]{4}{*}{ Annonaceae } & Annona coriacea Mart. & árvore & & $\mathrm{X}$ \\
\hline & Annona crassiflora Mart. & árvore & $\mathrm{X}$ & \\
\hline & Annona dioica Mart. & árvore & & $\mathrm{X}$ \\
\hline & Xylopia aromatica (Lam.) Mart. & árvore & $\mathrm{X}$ & $\mathrm{X}$ \\
\hline Apocynaceae & Aspidosperma tomentosum Mart. & árvore & $\mathrm{X}$ & $\mathrm{X}$ \\
\hline Araliaceae & Didymopanax vinosum (Cham. \& Schltdl.) March. & árvore & $\mathrm{X}$ & $\mathrm{X}$ \\
\hline \multirow[t]{2}{*}{ Arecaceae } & Attalea geraensis B. Rodr. & palmeira acaule & $\mathrm{X}$ & $\mathrm{X}$ \\
\hline & Butia paraguayensis (B. Rodr.) Bailey & palmeira acaule & & $\mathrm{X}$ \\
\hline \multirow[t]{6}{*}{ Asteraceae } & Baccharis dracunculifolia DC. & arbusto & & $\mathrm{X}$ \\
\hline & Blainvillea $\mathrm{sp}$. & erva & & $\mathrm{X}$ \\
\hline & Eupatorium sp. & erva & & $\mathrm{X}$ \\
\hline & Gochnatia lucida Cabrera & árvore & $\mathrm{X}$ & $\mathrm{X}$ \\
\hline & Piptocarpha rotundifolia (Less.) Baker & árvore & $\mathrm{X}$ & \\
\hline & Vernonia rubriramea Mart. ex DC. & árvore & & $\mathrm{X}$ \\
\hline \multirow[t]{4}{*}{ Bignoniaceae } & Arrabidaea brachypoda (DC.) Bur. & arbusto & & $\mathrm{X}$ \\
\hline & Jacaranda caroba (Vell.) DC. & arbusto & & $\mathrm{X}$ \\
\hline & Tabebuia ochracea (Cham.) Standl. & árvore & $\mathrm{X}$ & $\mathrm{X}$ \\
\hline & Zeyhera digitalis (Vell.) Hoehne & arbusto & & $\mathrm{X}$ \\
\hline Bombacaceae & Eriotheca gracilipes (Schum.) Robyns & árvore & $\mathrm{X}$ & \\
\hline Bromeliaceae & Bromelia balansae $\mathrm{Mez}$ & erva rosulada & & $\mathrm{X}$ \\
\hline \multirow[t]{2}{*}{ Caesalpiniaceae } & Dimorphandra mollis Benth. & árvore & $\mathrm{X}$ & \\
\hline & Senna rugosa (G.Don.) H.S. Irwin \& Barneby & arbusto & & $\mathrm{X}$ \\
\hline Caryocaraceae & Caryocar brasiliense Cambess. & árvore & & $\mathrm{X}$ \\
\hline \multirow[t]{3}{*}{ Clusiaceae } & Kielmeyera coriacea (Spreng.) Mart. & árvore & $\mathrm{X}$ & \\
\hline & Kielmeyera rubriflora A. St.-Hil. & árvore & $\mathrm{X}$ & \\
\hline & Kielmeyera variabilis Mart. & arbusto & $\mathrm{X}$ & $\mathrm{X}$ \\
\hline Connaraceae & Connarus suberosus Planch. & árvore & $\mathrm{X}$ & $\mathrm{X}$ \\
\hline Dilleniaceae & Davilla eliptica A. St.-Hil. & arbusto & & $\mathrm{X}$ \\
\hline Ebenaceae & Diospyros hispida DC. & árvore & $\mathrm{X}$ & $\mathrm{X}$ \\
\hline \multirow[t]{4}{*}{ Erythroxylaceae } & Erythroxylum campestre A. St.-Hil. & arbusto & & $\mathrm{X}$ \\
\hline & Erythroxylum suberosum A. St.-Hil. & árvore & $\mathrm{X}$ & $\mathrm{X}$ \\
\hline & Erythroxylum tortuosum Mart. & árvore & $\mathrm{X}$ & $\mathrm{X}$ \\
\hline & Erythroxylum cuneifolium Poepp. ex O.E. Schulz & árvore & $\mathrm{X}$ & $\mathrm{X}$ \\
\hline \multirow[t]{3}{*}{ Euphorbiaceae } & Pera glabrata (Schott.) Baill. & árvore & $\mathrm{X}$ & $\mathrm{X}$ \\
\hline & Pera obovata Baill. & árvore & $\mathrm{X}$ & \\
\hline & Sapium marginatum M. Arg. & subarbusto & & $\mathrm{X}$ \\
\hline \multirow[t]{3}{*}{ Fabaceae } & Acosmium subelegans (Mohl.) Yakovlev & árvore & $\mathrm{X}$ & $\mathrm{X}$ \\
\hline & Dalbergia miscolobium Benth. & árvore & $\mathrm{X}$ & $\mathrm{X}$ \\
\hline & Machaerium acutifolium Vogel & árvore & $\mathrm{X}$ & \\
\hline Flacourtiaceae & Casearia sylvestris $\mathrm{Sw}$. & árvore & & $\mathrm{X}$ \\
\hline Hippocrateaceae & Salacia campestris Walp. & subarbusto & & $\mathrm{X}$ \\
\hline Lamiaceae & Lippia balansae Brig. & arbusto & & $\mathrm{X}$ \\
\hline Lauraceae & Ocotea pulchella Mart. & árvore & $\mathrm{X}$ & $\mathrm{X}$ \\
\hline Malpiguiaceae & Banisteriopsis campestris (A. Juss.) B. Gates & subarbusto & & $\mathrm{X}$ \\
\hline
\end{tabular}


Tabela 1. (continuação).

\begin{tabular}{|c|c|c|c|c|}
\hline FAMÍLIA & ESPÉCIE & HÁBITO & $\mathrm{S}$ & I \\
\hline & Byrsonima coccolobifolia Kunth & árvore & $\mathrm{X}$ & $\mathrm{X}$ \\
\hline & Byrsonima intermedia A. Juss. & arbusto & & $\mathrm{X}$ \\
\hline \multirow[t]{4}{*}{ Melastomataceae } & Leandra involucrata DC. & árvore & $\mathrm{X}$ & \\
\hline & Leandra lacunosa Cogn. & arbusto & & $\mathrm{X}$ \\
\hline & Miconia albicans (Sw.) Triana & árvore & $\mathrm{X}$ & $\mathrm{X}$ \\
\hline & Miconia rubiginosa (Bonpl.) DC. & árvore & $\mathrm{X}$ & $\mathrm{X}$ \\
\hline Menispermaceae & Cissampelos ovalifolia DC. & subarbusto & & $\mathrm{X}$ \\
\hline \multirow[t]{3}{*}{ Mimosaceae } & Anadenanthera falcata (Benth.) Speg. & árvore & $\mathrm{X}$ & \\
\hline & Stryphnodendron adstringens (Mart.) Coville & árvore & $\mathrm{X}$ & \\
\hline & Stryphnodendron obovatum Benth & árvore & $X$ & $\mathrm{X}$ \\
\hline \multirow[t]{2}{*}{ Myrsinaceae } & Rapanea guianensis Aubl. & árvore & $\mathrm{X}$ & $\mathrm{X}$ \\
\hline & Rapanea umbellata Mart. & árvore & $\mathrm{X}$ & $\mathrm{X}$ \\
\hline \multirow[t]{11}{*}{ Myrtaceae } & Calyptranthes concinna DC. & árvore & & $\mathrm{X}$ \\
\hline & Campomanesia adamantium (Camb.) O. Berg. & arbusto & $\mathrm{X}$ & $\mathrm{X}$ \\
\hline & Campomanesia pubescens (DC.) O. Berg. & arbusto & & $\mathrm{X}$ \\
\hline & Eugenia aurata $\mathrm{O}$. Berg & árvore & & $\mathrm{X}$ \\
\hline & Eugenia bimarginata $\mathrm{DC}$. & arbusto & & $\mathrm{X}$ \\
\hline & Eugenia dysenterica DC. & subarbusto & & $\mathrm{X}$ \\
\hline & Eugenia pyriformis Cambess. & árvore & & $\mathrm{X}$ \\
\hline & Myrcia albo-tomentosa Cambess. & árvore & $\mathrm{X}$ & \\
\hline & Myrcia lingua (O. Berg.) Mattos & árvore & $\mathrm{X}$ & $\mathrm{X}$ \\
\hline & Psidium cinereum DC. & arbusto & & $\mathrm{X}$ \\
\hline & Psidium luridum (Spreng.) Bur. & arbusto & & $\mathrm{X}$ \\
\hline Ochnaceae & Ouratea spectabilis (Mart.) Endl. & árvore & $\mathrm{X}$ & $\mathrm{X}$ \\
\hline \multirow[t]{2}{*}{ Rubiaceae } & Alibertia concolor (Cham.) K. Schum. & subarbusto & & $\mathrm{X}$ \\
\hline & Tocoyena formosa (Cham. \& Schlecht.) K. Schum. & arbusto & $\mathrm{X}$ & $\mathrm{X}$ \\
\hline Rutaceae & Zanthoxylum rhoifolium Lam. & árvore & $\mathrm{X}$ & \\
\hline Sapindaceae & Talisia pygmaea Radlk. & subarbusto & & $\mathrm{X}$ \\
\hline Sapotaceae & Pouteria torta (Mart.) Radlk. & árvore & $X$ & \\
\hline Solanaceae & Solanum inaequale Vell. & árvore & & $\mathrm{X}$ \\
\hline \multirow[t]{2}{*}{ Styracaceae } & Styrax camporum Pohl & árvore & $\mathrm{X}$ & \\
\hline & Styrax ferrugineus Nees \& Mart. & árvore & $\mathrm{X}$ & \\
\hline Verbenaceae & Aegiphila lhotzkyana Cham. & árvore & $\mathrm{X}$ & $\mathrm{X}$ \\
\hline Vochysiaceae & Qualea grandiflora Mart. & árvore & $X$ & \\
\hline
\end{tabular}

A densidade do estrato arbóreo, obtida pelo método de parcelas, foi de $1150 \mathrm{ind} / \mathrm{ha}$. A estimativa de densidade obtida pelo método de quadrantes, dentro da mesma área amostral, gerou um valor de 869 ind/ha, $24,4 \%$ inferior ao valor encontrado pelo método de parcelas. A subestimativa da densidade pelo método de quadrantes era esperada, em concordância com as ponderações de
Cottam et al. (1953), uma vez que os indivíduos distribuem-se de forma agregada na área amostral $(\mathrm{P}=4,26)$.

$\mathrm{Na}$ análise de distribuição espacial das populações, encontraram-se 12 espécies com distribuição agregada, 7 espécies com distribuição aleatória e 19 espécies com distribuição regular. As espécies com maior índice de agregação foram Pouteria torta, Aspidosperma 
Tabela 2. Espécies presentes no estrato superior da vegetação de cerrado stricto sensu, Brotas, SP, em ordem decrescente de VI (valor de importância). DA: densidade absoluta; DR: densidade relativa; FA: freqüência absoluta; FR: freqüência relativa; DoA: dominância absoluta; DoR: dominância relativa; VC: valor de cobertura; P: índice de agregação de Payandeh.

\begin{tabular}{|c|c|c|c|c|c|c|c|c|c|}
\hline Espécie & $\begin{array}{c}\text { DA } \\
\text { (ind/ha) }\end{array}$ & $\begin{array}{l}\mathrm{DR} \\
(\%)\end{array}$ & $\begin{array}{l}\text { FA } \\
(\%)\end{array}$ & $\begin{array}{l}\text { FR } \\
(\%)\end{array}$ & $\begin{array}{c}\text { DoA } \\
\left(\mathrm{m}^{2} / \mathrm{ha}\right)\end{array}$ & $\begin{array}{c}\text { DoR } \\
(\%)\end{array}$ & VI & $\mathrm{VC}$ & $\mathrm{P}^{*}$ \\
\hline Acosmium subelegans & 218 & 18,96 & 60 & 9,80 & 1,15 & 16,03 & 44,79 & 34,99 & 3,06 \\
\hline Aspidosperma tomentosum & 170 & 14,78 & 48 & 7,84 & 0,57 & 7,89 & 30,51 & 22,67 & 4,40 \\
\hline Eriotheca gracilipes & 88 & 7,65 & 38 & 6,21 & 0,75 & 10,45 & 24,31 & 18,10 & 2,58 \\
\hline Styrax ferrugineus & 28 & 2,43 & 20 & 3,27 & 0,70 & 9,75 & 15,46 & 12,19 & 1,46 \\
\hline Tabebuia ochracea & 72 & 6,26 & 36 & 5,88 & 0,24 & 3,28 & 15,42 & 9,54 & 2,27 \\
\hline Erythroxylum tortuosum & 54 & 4,70 & 36 & 5,88 & 0,25 & 3,55 & 14,13 & 8,25 & 1,38 \\
\hline Stryphnodendron obovatum & 44 & 3,83 & 36 & 5,88 & 0,22 & 3,09 & 12,80 & 6,92 & 0,94 \\
\hline Piptocarpha rotundifolia & 44 & 3,83 & 26 & 4,25 & 0,28 & 3,85 & 11,92 & 7,68 & 1,96 \\
\hline Ocotea pulchella & 36 & 3,13 & 28 & 4,58 & 0,28 & 3,96 & 11,66 & 7,09 & 1,45 \\
\hline Annona crassiflora & 28 & 2,43 & 22 & 3,59 & 0,28 & 3,94 & 9,96 & 6,37 & 1,17 \\
\hline Didymopanax vinosum & 32 & 2,78 & 28 & 4,58 & 0,16 & 2,25 & 9,61 & 5,03 & 0,95 \\
\hline Ouratea spectabilis & 24 & 2,09 & 22 & 3,59 & 0,21 & 2,96 & 8,65 & 5,05 & 0,95 \\
\hline Rapanea guianensis & 34 & 2,96 & 14 & 2,29 & 0,18 & 2,48 & 7,73 & 5,44 & 4,39 \\
\hline Aegiphila lhotzkyana & 24 & 2,09 & 20 & 3,27 & 0,12 & 1,70 & 7,05 & 3,78 & 1,12 \\
\hline Miconia rubiginosa & 22 & 1,91 & 14 & 2,29 & 0,20 & 2,84 & 7,04 & 4,76 & 1,91 \\
\hline Kielmeyera variabilis & 28 & 2,43 & 14 & 2,29 & 0,14 & 1,97 & 6,69 & 4,40 & 2,78 \\
\hline Rapanea umbellata & 24 & 2,09 & 12 & 1,96 & 0,16 & 2,25 & 6,30 & 4,34 & 2,48 \\
\hline Xylopia aromatica & 20 & 1,74 & 14 & 2,29 & 0,07 & 0,97 & 5,00 & 2,71 & 1,43 \\
\hline Qualea grandiflora & 16 & 1,39 & 6 & 0,98 & 0,17 & 2,41 & 4,78 & 3,80 & 3,15 \\
\hline Pera glabrata & 10 & 0,87 & 10 & 1,63 & 0,16 & 2,17 & 4,67 & 3,04 & 0,92 \\
\hline Pouteria torta & 26 & 2,26 & 4 & 0,65 & 0,12 & 1,65 & 4,56 & 3,91 & 6,41 \\
\hline Erythroxylum suberosum & 14 & 1,22 & 12 & 1,96 & 0,04 & 0,61 & 3,79 & 1,83 & 1,17 \\
\hline Attalea geraensis & 4 & 0,35 & 4 & 0,65 & 0,16 & 2,30 & 3,30 & 2,64 & 0,98 \\
\hline Machaerium acutifolium & 10 & 0,87 & 10 & 1,63 & 0,04 & 0,50 & 3,00 & 1,37 & 0,92 \\
\hline Diospyros hispida & 8 & 0,70 & 8 & 1,31 & 0,06 & 0,88 & 2,88 & 1,57 & 0,94 \\
\hline Connarus suberosus & 8 & 0,70 & 8 & 1,31 & 0,03 & 0,44 & 2,44 & 1,13 & 0,94 \\
\hline Pera obovata & 6 & 0,52 & 6 & 0,98 & 0,04 & 0,57 & 2,07 & 1,09 & 0,96 \\
\hline Miconia albicans & 6 & 0,52 & 6 & 0,98 & 0,02 & 0,31 & 1,81 & 0,83 & 0,96 \\
\hline Dimorphandra mollis & 4 & 0,35 & 4 & 0,65 & 0,05 & 0,69 & 1,70 & 1,04 & 0,98 \\
\hline Stryphnodendron adstringens & 4 & 0,35 & 4 & 0,65 & 0,04 & 0,56 & 1,56 & 0,91 & 0,98 \\
\hline Kielmeyera rubriflora & 4 & 0,35 & 2 & 0,33 & 0,06 & 0,84 & 1,52 & 1,19 & 2,00 \\
\hline Zanthoxyllum rhoifolium & 4 & 0,35 & 4 & 0,65 & 0,04 & 0,49 & 1,49 & 0,84 & 0,98 \\
\hline Myrcia albo-tomentosa & 4 & 0,35 & 4 & 0,65 & 0,02 & 0,32 & 1,32 & 0,67 & 0,98 \\
\hline Byrsonima coccolobifolia & 4 & 0,35 & 4 & 0,65 & 0,02 & 0,23 & 1,23 & 0,58 & 0,98 \\
\hline Dalbergia miscolobium & 4 & 0,35 & 4 & 0,65 & 0,01 & 0,19 & 1,19 & 0,53 & 0,98 \\
\hline Gochnatia lucida & 4 & 0,35 & 4 & 0,65 & 0,01 & 0,16 & 1,16 & 0,51 & 0,98 \\
\hline Leandra involucrata & 2 & 0,17 & 2 & 0,33 & 0,05 & 0,64 & 1,14 & 0,82 & \\
\hline Campomanesia adamantium & 4 & 0,35 & 4 & 0,65 & 0,01 & 0,13 & 1,13 & 0,48 & 0,98 \\
\hline Styrax camporum & 4 & 0,35 & 4 & 0,65 & 0,01 & 0,11 & 1,11 & 0,46 & 0,98 \\
\hline Tocoyena formosa & 2 & 0,17 & 2 & 0,33 & 0,01 & 0,20 & 0,70 & 0,37 & \\
\hline Kielmeyera coriacea & 2 & 0,17 & 2 & 0,33 & 0,01 & 0,18 & 0,68 & 0,35 & \\
\hline Anadenanthera falcata & 2 & 0,17 & 2 & 0,33 & 0,01 & 0,08 & 0,58 & 0,25 & \\
\hline Myrcia lingua & 2 & 0,17 & 2 & 0,33 & 0,01 & 0,08 & 0,58 & 0,25 & \\
\hline Erythroxylum cuneifolium & 2 & 0,17 & 2 & 0,33 & 0,01 & 0,05 & 0,56 & 0,23 & \\
\hline Total & 1150 & & 612 & & 7,18 & & 300 & 200 & 4,26 \\
\hline
\end{tabular}

* $\mathrm{P}>1,5$ - distribuição agregada; $\mathrm{P}$ de 1,0 a 1,5 - distribuição aleatória; $\mathrm{P}<1,0$ - distribuição regular. 
tomentosum e Rapanea guianensis. A alta agregação dos indivíduos da comunidade como um todo e das populações de algumas espécies está, provavelmente, associada ao modo de regeneração dos indivíduos, após incêndios ou corte. A maioria das espécies de cerrado pode regenerar-se facilmente por brotação de estruturas subterrâneas, muitas vezes gerando vários indivíduos geneticamente idênticos, a partir de um único indivíduo pré-existente.

As árvores do estrato superior são de pequeno porte, com altura máxima de $7 \mathrm{~m}$ e diâmetro máximo de $32 \mathrm{~cm}$, resultando em baixa cobertura de copas $(19,6 \%)$ e área basal de apenas 7,2 $\mathrm{m}^{2} /$ ha. Estes valores correspondem à classificação fisionômica da vegetação de "cerrado típico", segundo Ribeiro et al. (1985), mais comumente denominada cerrado stricto sensu. Em outras áreas de cerrado stricto sensu, Goodland (1979) encontrou área basal de $16,2 \mathrm{~m}^{2} / \mathrm{ha}$, Ribeiro et al. (1985) obtiveram 9,65 $\mathrm{m}^{2} /$ ha e Sambuichi \& Eiten (2000) encontraram $11,7 \mathrm{~m}^{2} / \mathrm{ha}$.

Analisando-se as espécies amostradas quanto à sua importância relativa no estrato superior, em ordem decrescente, destacaram-se as espécies: Acosmium subelegans, Aspidosperma tomentosum, Eriotheca gracilipes, Styrax ferrugineus e Tabebuia ochracea.

$\mathrm{O}$ índice de diversidade $\left(\mathrm{H}^{\prime}\right)$ obtido para o estrato superior foi de 3,02 (equabilidade de $80 \%$ ), valor menor que o obtido em outras áreas de cerrado da região. Giannotti (1988) obteve o maior valor: 3,74 e Durigan et al. (1994) obtiveram o menor valor: 3,08, em áreas de cerrado no município de Itirapina, sendo este último estudo em área submetida a incêndios freqüentes.

No estrato inferior, a densidade de indivíduos foi de $10.220 \mathrm{ind} / \mathrm{ha}$, pertencentes a 61 espécies e 32 famílias (Tab. 3). A comunidade como um todo apresenta-se agregada também no estrato inferior $(\mathrm{P}=3,89)$. Analisando-se separadamente as populações no estrato inferior, encontraram-se 13 espécies com distribuição agregada, 13 com distribuição aleatória e 19 com distribuição regular. As espécies com agregação mais forte foram Bromelia balansae, Rapanea umbellata e Ocotea pulchella. O índice de diversidade $\left(\mathrm{H}^{\prime}\right)$ obtido foi de 3,32 (equabilidade de $81 \%$ ). As espécies que se destacaram no estrato inferior, em ordem decrescente de densidade relativa, foram: Campomanesia adamantium, Bromelia balansae, Attalea geraensis, Xylopia aromatica e Ocotea pulchella.

$\mathrm{Na}$ análise de similaridade entre os dois estratos, encontrou-se $35,1 \%$, pelo índice de Jaccard, valor considerado elevado para comparação entre áreas, mas inferior ao esperado para dois estratos da vegetação de cerrado stricto sensu em um mesmo local.

A maior riqueza e diversidade encontradas no estrato inferior eram previsíveis para vegetação de cerrado stricto sensu, já que, naturalmente, muitas espécies de porte não arbóreo são amostradas apenas no estrato inferior. É surpreendente, no entanto, a ausência, no estrato inferior, de um número considerável de espécies (17), algumas das quais apresentam-se com alta densidade no estrato superior, como: Eriotheca gracilipes, Styrax ferrugineus, Piptocarpha rotundifolia e Annona crassiflora, entre outras. Em ambientes florestais, ou mesmo no cerradão, a ausência de espécies heliófitas no estrato inferior é compreensível. A regeneração dessas espécies ocorre em clareiras ou na borda dos fragmentos. Porém, a vegetação de cerrado strito sensu não apresenta condições microclimáticas distintas entre estratos, a ponto de impedir a regeneração das espécies arbóreas. Por alguma razão, provavelmente relacionada com a biologia reprodutiva destas espécies, não está ocorrendo, há algum tempo, o recrutamento de novos indivíduos à comunidade. Podem ser aventadas como causas possíveis, por exemplo, a ausência de polinizadores ou dispersores específicos, já que esta área tem sofrido fortes pressões de isolamento, tendo a leste a extensa área da Represa do Lobo (formada há cerca de 70 anos) e a oeste a Estação 
Tabela 3. Espécies vegetais amostradas no estrato inferior da vegetação de cerrado stricto sensu, Brotas, SP, em ordem decrescente de densidade relativa. DA: densidade absoluta; DR: densidade relativa; FA: freqüência absoluta; FR: freqüência relativa; P: índice de agregação de Payandeh.

\begin{tabular}{|c|c|c|c|c|c|}
\hline Espécie & $\begin{array}{c}\text { DA } \\
\text { (ind/ha) }\end{array}$ & $\begin{array}{l}\text { DR } \\
(\%)\end{array}$ & $\begin{array}{l}\text { FA } \\
(\%)\end{array}$ & $\begin{array}{l}\text { FR } \\
(\%)\end{array}$ & $\mathrm{P} *$ \\
\hline Campomanesia adamantium & 2260 & 22,11 & 56 & 9,38 & 3,57 \\
\hline Bromelia balansae & 820 & 8,02 & 12 & 2,01 & 13,27 \\
\hline Attalea geraensis & 600 & 5,87 & 42 & 7,04 & 1,09 \\
\hline Xylopia aromatica & 540 & 5,28 & 30 & 5,03 & 1,68 \\
\hline Ocotea pulchella & 440 & 4,31 & 14 & 2,35 & 4,47 \\
\hline Miconia albicans & 400 & 3,91 & 26 & 4,36 & 1,73 \\
\hline Rapanea umbellata & 380 & 3,72 & 14 & 2,35 & 4,61 \\
\hline Jacaranda caroba & 300 & 2,94 & 30 & 5,03 & 0,71 \\
\hline Gochnatia lucida & 240 & 2,35 & 22 & 3,69 & 0,95 \\
\hline Tabebuia ochracea & 240 & 2,35 & 18 & 3,02 & 1,46 \\
\hline Byrsonima intermedia & 220 & 2,15 & 16 & 2,68 & 1,54 \\
\hline Erythroxylum campestre & 220 & 2,15 & 10 & 1,68 & 3,76 \\
\hline Eupatorium sp & 200 & 1,96 & 18 & 3,02 & 1,02 \\
\hline Rapanea guianensis & 200 & 1,96 & 18 & 3,02 & 1,02 \\
\hline Senna rugosa & 160 & 1,57 & 10 & 1,68 & 1,88 \\
\hline Vernonia rubriramea & 160 & 1,57 & 12 & 2,01 & 1,37 \\
\hline Aspidosperma tomentosum & 160 & 1,57 & 8 & 1,34 & 2,64 \\
\hline Salacia campestris & 160 & 1,57 & 12 & 2,01 & 1,37 \\
\hline Acosmium subelegans & 160 & 1,57 & 16 & 2,68 & 0,86 \\
\hline Lippia balansae & 160 & 1,57 & 14 & 2,35 & 1,11 \\
\hline Aegiphila lhotzkyana & 140 & 1,37 & 14 & 2,35 & 0,88 \\
\hline Pera glabrata & 140 & 1,37 & 14 & 2,35 & 0,88 \\
\hline Miconia rubiginosa & 120 & 1,17 & 10 & 1,68 & 1,24 \\
\hline Talisia pygmaea & 120 & 1,17 & 8 & 1,34 & 1,58 \\
\hline Didymopanax vinosum & 100 & 0,98 & 10 & 1,68 & 0,92 \\
\hline Erythroxylum suberosum & 100 & 0,98 & 10 & 1,68 & 0,92 \\
\hline Myrcia lingua & 100 & 0,98 & 8 & 1,34 & 1,33 \\
\hline Psidium luridum & 100 & 0,98 & 8 & 1,34 & 1,33 \\
\hline Byrsonima coccolobifolia & 80 & 0,78 & 6 & 1,01 & 1,45 \\
\hline Arrabidaea brachypoda & 80 & 0,78 & 6 & 1,01 & 1,45 \\
\hline Baccharis dracunculifolia & 80 & 0,78 & 8 & 1,34 & 0,94 \\
\hline Kielmeyera variabilis & 80 & 0,78 & 6 & 1,01 & 1,45 \\
\hline Calyptranthes concinna & 60 & 0,59 & 4 & 0,67 & 1,64 \\
\hline Eugenia aurata & 60 & 0,59 & 6 & 1,01 & 0,96 \\
\hline Leandra lacunosa & 60 & 0,59 & 6 & 1,01 & 0,96 \\
\hline Psidium cinereum & 60 & 0,59 & 6 & 1,01 & 0,96 \\
\hline Stryphnodendron obovatum & 60 & 0,59 & 6 & 1,01 & 0,96 \\
\hline Alibertia concolor & 60 & 0,59 & 6 & 1,01 & 0,96 \\
\hline
\end{tabular}


Tabela 3. (continuação).

\begin{tabular}{lrrrrr}
\hline Espécie & $\begin{array}{r}\text { DA } \\
\text { (ind/ha) }\end{array}$ & $\begin{array}{r}\text { DR } \\
(\%)\end{array}$ & $\begin{array}{r}\text { FA } \\
(\%)\end{array}$ & $\begin{array}{r}\text { FR } \\
(\%)\end{array}$ & P* \\
\hline Annona dioica & 40 & 0,39 & 4 & 0,67 & 0,98 \\
Butia paraguayensis & 40 & 0,39 & 4 & 0,67 & 0,98 \\
Caryocar brasiliense & 40 & 0,39 & 2 & 0,34 & 2,00 \\
Dalbergia miscolobium & 40 & 0,39 & 4 & 0,67 & 0,98 \\
Erythroxylum tortuosum & 40 & 0,39 & 4 & 0,67 & 0,98 \\
Eugenia dysenterica & 40 & 0,39 & 4 & 0,67 & 0,98 \\
Casearia sylvestris & 40 & 0,39 & 4 & 0,67 & 0,98 \\
Annona coriacea & 20 & 0,20 & 2 & 0,34 & \\
Blainvillea sp & 20 & 0,20 & 2 & 0,34 & \\
Banisteriopsis campestris & 20 & 0,20 & 2 & 0,34 & \\
Campomanesia pubescens & 20 & 0,20 & 2 & 0,34 & \\
Cissampelos ovalifolia & 20 & 0,20 & 2 & 0,34 & \\
Connarus suberosus & 20 & 0,20 & 2 & 0,34 & \\
Davilla eliptica & 20 & 0,20 & 2 & 0,34 & \\
Diospyros hispida & 20 & 0,20 & 2 & 0,34 & \\
Erythroxylum cuneifolium & 20 & 0,20 & 2 & 0,34 & \\
Eugenia bimarginata & 20 & 0,20 & 2 & 0,34 & \\
Eugenia pyriformis & 20 & 0,20 & 2 & 0,34 & \\
Ouratea spectabilis & 20 & 0,20 & 2 & 0,34 & \\
Sapium marginatum & 20 & 0,20 & 2 & 0,34 & \\
Solanum inaequale & 20 & 0,20 & 2 & 0,34 & \\
Tocoyena formosa & 0,20 & 2 & 0,34 & \\
Zeyhera digitalis & 0220 & & 2 & 0,34 & \\
\hline TOTAL & 20 & 0,20 & & & \\
\hline P & 20,89 \\
\hline
\end{tabular}

* P > 1,5 - distribuição agregada; $\mathrm{P}$ de 1,0 a 1,5 - distribuição aleatória; $\mathrm{P}<1,0$ - distribuição regular.

Ecológica de Itirapina, em que predominam as fisionomias de campo úmido e campo cerrado, com poucas espécies arbóreas.

Na ausência de recrutamento, a morte dos adultos existentes poderá significar extinção local destas espécies, resultando em perda considerável de diversidade a médio prazo, caso não ocorra o ingresso de novas espécies na comunidade.

\section{Referências bibliográficas}

Batalha, M.A. \& Mantovani, W. 1999. Chave de identificação baseada em caracteres vegetativos para as espécies vasculares do cerrado na ARIE Pé-de-Gigante (Santa Rita do Passa Quatro, SP). Revista do
Instituto Florestal 11(2):137-158.

Borgonovi, M. \& Chiarini, J.V. 1965. Cobertura vegetal do Estado de São Paulo. I - Levantamento por fotointerpretação das áreas cobertas com cerrado, cerradão e campo, em 1962. Bragantia 24:159-172.

Canfield, R. 1941. Application of line interception in sampling range vegetation. Journal of Forestry 39:388-394.

Castro, A.A.J.F. 1987. Florística e fitossociologia de um cerrado marginal brasileiro, Parque Estadual de Vaçununga - SP. Dissertação de Mestrado. Instituto de Biologia-Universidade Estadual de Campinas, Campinas.

Cesar, O.; Pagano, S.N.; Leitão Filho, H.F.; Monteiro, R.; Silva, O.A.; Marinis, G. \& Shepherd, G.O. 1988. Estrutura fitossociológica do estrato arbóreo de uma 
área de vegetação de cerrado no município de Corumbataí (Estado de São Paulo). Naturalia 13:91-101.

Chiarini, J.V. \& Coelho, A.G.S. 1969. Cobertura vegetal natural e áreas reflorestadas do Estado de São Paulo. Secretaria da Agricultura do Estado de São Paulo. Instituto Agronômico. Boletim 193. Campinas, 28p.

Cottam, G. \& Curtis, J.T. 1956. The use of distance measures in phytosociological sampling. Ecology 37:451-460.

Cottam, G.; Curtis, J.T. \& Hale, B.W. 1953. Some sampling characteristics of a population of randomly dispersed individuals. Ecology 34:741-747.

Durigan, G.; Bacic, M.C.; Franco, G.A.D.C. \& Siqueira, M.F. 1999. Inventário florístico do cerrado na Estação Ecológica de Assis, SP. Hoehnea 26:149-172.

Durigan, G.; Leitão Filho, H.F. \& Rodrigues, R.R. 1994. Phytosociology and structure of a frequently burnt cerrado vegetation in SE-Brazil. Flora 189: $153-160$

Eiten, G. 1972. The cerrado vegetation of Brazil. Botanical Review 38(2):201-341.

Freire, O.; Gimenes, R.J.; Pessoti, J.E. \& Carraro, E. 1980. Solos da Bacia do Broa. Depto. de Ciências Biológicas. Universidade Federal de São Carlos. São Carlos. 85p.

Giannotti, E. 1988. Composição florística e estrutura fitossociológica da vegetação de cerrado e de transição entre cerrado e mata ciliar da Estação Experimental de Itirapina (SP). Dissertação de Mestrado. Instituto de Biologia. Universidade Estadual de Campinas. Campinas.

Goodland, R. 1979. Análise ecológica da vegetação do cerrado. Pp.61-171. In: Goodland, R. \& Ferri, M.G. (eds.) Ecologia do Cerrado. Itatiaia, Belo Horizonte.

Kronka, F.J.N.; Nalon, M.A.; Matsukuma, C.K.; Pavão, M.; Guillaumon, J.R.; Cavalli, A.C.; Giannotti, E.; Iwane, M.S.S.; Lima, L.M.P.R.; Montes, J.; Del Cali, I.H. \& Haack, P.G. 1998. Áreas de domínio do cerrado no Estado de São Paulo. São Paulo. Secretaria de Estado do Meio Ambiente, Instituto Florestal.

Leitão Filho, H.F. 1992. A flora arbórea dos cerrados do Estado de São Paulo. Hoehnea 19(1/2):151-163.

Magurran, A.E. 1988. Ecological diversity and its measurement. Princeton. Princeton University Press.

Mantovani, W. 1987. Análise florística do estrato herbáceo-subarbustivo do cerrado na Reserva Bioló- gica de Moji-Guaçu e em Itirapina. Tese de Doutorado. Universidade Estadual de Campinas. Campinas. Mantovani, W.; Leitão Filho, H.F. \& Martins, F.R. 1985. Chave baseada em caracteres vegetativos para identificação de espécies lenhosas do cerrado da Reserva Biológica de Moji-Guaçu, Estado de São Paulo. Hoehnea 12:35-56.

Messina, R. 1998. Elaboração e implantação de um modelo de recomposição ciliar às margens da Represa do Lobo, Itirapina, SP. Dissertação de Mestrado. Escola de Engenharia de São Carlos. Universidade de São Paulo, São Carlos.

Müller-Dombois, D. \& Ellenberg, H. 1974. Aims and methods in vegetation ecology. New York. John Wiley and Sons.

Pagano, S.N.; Cesar, O. \& Leitão Filho, H.F. 1989. Estrutura fitossociológica do estrato arbustivo-arbóreo da vegetação de cerrado da Área de Proteção Ambiental (APA) de Corumbataí - Estado de São Paulo. Revista Brasileira de Biologia 49:49-59.

Payandeh, B. 1970. Comparison of methods for assessing spacial distribution of trees. Forest Science 16(3):312-317.

Pielou, E.C. 1969. An introduction to mathematical ecology. Wiley. New York.

Pielou, E.C. 1975. Ecological diversity. New York. John Wiley and Sons.

Ribeiro, J.F.; Silva, J.C.S. \& Batmanian, G.J. 1985. Fitossociologia de tipos fisionômicos de cerrado em Planaltina-DF. Revista Brasileira de Botânica 8:131-142.

Sambuichi, R.H.R. \& Eiten, G. 2000. Fitossociologia da camada lenhosa de um cerrado em Brasília, DF. Boletim do Herbário Ezechias Paulo Heringer 5:62-87.

São Paulo, Secretaria de Estado do Meio Ambiente. 1997. Bases para a conservação e uso sustentável das áreas de cerrado do Estado de São Paulo. São Paulo. (Série PROBIO/SP).

Serra Filho, R.; Cavalli, A.C.; Guillaumon, J.R.; Chiarini, J.V.; Nogueira, F.P.; Ivancko, C.M.A.M.; Barbieri, J.L.; Donzelli, P.L.; Coelho, A.G.S. \& Bittencourt, L. 1974. Levantamento da cobertura natural e do reflorestamento no Estado de São Paulo. Boletim Técnico do Instituto Florestal, São Paulo, 11.

Toledo Filho, D.V.; Leitão Filho, H.F. \& Shepherd, G. 1989. Estrutura fitossociológica da vegetação de cerrado em Mogi-Mirim (SP). Revista do Instituto Florestal 1(2):1-11. 